\title{
A CANONICAL FORM FOR PLANAR FAREY SETS
}

\author{
NORMAN RICHERT
}

\begin{abstract}
Asmus Schmidt has developed a new algorithm for generating Gaussian rational approximations to complex irrational numbers. The method is based on the structure of a certain collection of planar sets. Schmidt suggested an inductive proof for the structure theorem without publishing the details. The present paper presents a proof, which is not inductive in character.
\end{abstract}

In a 1975 Acta Mathematica paper [3], Asmus Schmidt developed a new method for generating Gaussian rational approximations to complex irrational numbers. The approximations correspond to decreasing sequences of planar sets, which can be viewed as converging to a number $\xi$. These sets, which are called Farey sets, in turn correspond to complex unimodular transformations. Each Farey set has a canonical representation in terms of a composition of elements of a special set of seven unimodular maps. Schmidt's method can be viewed as analogous to the classical continued fraction algorithm for real numbers, with a sequence of the seven special maps corresponding to the usual sequence of partial quotients. Each complex irrational number has at most 2 sequences of maps associated with it. These sequences (called chains) are periodic if $\xi$ is quadratic and can be used to find the fundamental solution to the Pellian equation $X^{2}-D Y^{2}= \pm 1$, to mention but two analogies to continued fractions.

This paper contains a new proof [2] of the canonical form for Farey sets, on which Schmidt's method depends. The relevant definitions are introduced first, together with four fundamental lemmas.

Let $G$ be the set of unimodular transformations, i.e. complex bilinear maps of the form $m: z \rightarrow(a z+b) /(c z+d)$, where $a, b, c, d \in \mathbf{Z}[i]$ and det $m=a d-b c \in$ $\mathcal{Q}=\{ \pm 1, \pm i\}$. Let $G_{p}$ and $G_{i}$ partition $G$ into maps with $\operatorname{det} m= \pm 1$ and det $m= \pm i$ respectively. Let the closed upper half-plane be denoted by $\mathcal{T}$, let $\mathcal{T}^{*}$ denote the set

$$
\mathcal{T}^{*}=\left\{z: 0 \leqslant \operatorname{Re} z \leqslant 1, \operatorname{Im} z>0, N\left(z-\frac{1}{2}\right)>\frac{1}{4}\right\},
$$

and let $\overline{\mathcal{T}}$ and $\overline{\sigma^{*}}$ be the conjugate sets.

For each $m \in G_{p}$, the circular Farey set $F(m)$ is one of the two sets $m(\mathcal{T})$ or $m(\overline{\mathfrak{T}})$. The choice is made so that $F(m)$ is bounded if $m(\mathbf{R})$ is a circle and is bounded below if $m(\mathbf{R})$ is a line. Recall that $G$ maps circles to circles, where lines are considered to be degenerate circles. It is not hard to show that if $m(\mathbf{R})$ is a line, then it is horizontal, and an integral distance from $\mathbf{R}$.

Received by the editors February 13, 1980. Presented at the January 1981 Annual Meeting in San Francisco.

1980 Mathematics Subject Classification. Primary 10F20.

(c) 1981 American Mathematical Society $0002-9939 / 81 / 0000-0458 / \$ 02.00$ 
For each $m \in G_{i}$, the triangular Farey set $F(m)$ is one of the two sets $m\left(\mathcal{T}^{*}\right)$ or $m\left(\overline{\mathcal{J}}^{*}\right)$, where the choice is made so that $F(m)$ is inside the bounded region defined by $m(\mathbf{R})$ if $m(\mathbf{R})$ is a circle, and does not cross the imaginary axis if $m(\mathbf{R})$ is a line (such lines are vertical, at an integral distance from the imaginary axis).

Let $\mathscr{F}$ denote the collection of all circular and triangular Farey sets. It might be noted that all Ford circles and mesh triangles [1] are contained in $\mathscr{F}$. It is a remarkable fact, and crucial to Schmidt's method, that there is a canonical representation for elements of $\mathscr{F}$. This representation is based on the seven unimodular maps in $t_{F}=\left\{v_{1}, v_{2}, v_{3}, e_{1}, e_{2}, e_{3}, c\right\}$, where the corresponding matrices are

$$
\begin{array}{cc}
V_{1}=\left(\begin{array}{ll}
1 & i \\
0 & 1
\end{array}\right), & V_{2}=\left(\begin{array}{cc}
0 & 1 \\
-i & 1
\end{array}\right), \quad V_{3}=\left(\begin{array}{cc}
1-i & i \\
-1 & 1+i
\end{array}\right), \\
E_{1}=\left(\begin{array}{cc}
1 & 0 \\
1-i & i
\end{array}\right), & E_{2}=\left(\begin{array}{cc}
1 & -1+i \\
0 & i
\end{array}\right), \quad E_{3}=\left(\begin{array}{ll}
i & 0 \\
0 & 1
\end{array}\right), \\
C & =\left(\begin{array}{cc}
1 & -1+i \\
1-i & i
\end{array}\right) .
\end{array}
$$

The Farey sets $F(t)$ for $t \in t_{F}$ are denoted $\Upsilon_{1}, \mathscr{V}_{2}, \mathscr{V}_{3}, \mathcal{E}_{1}, \mathcal{E}_{2}, \mathcal{E}_{3}$, and $\mathcal{C}$ respectively. These sets are illustrated in Figure 1. Note, for example, that $\mathcal{V}_{1}=F\left(v_{1}\right)=$ $v_{1}(\mathcal{T})$ and $\varepsilon_{3}=F\left(e_{3}\right)=e_{3}\left(\mathcal{T}^{*}\right)$.

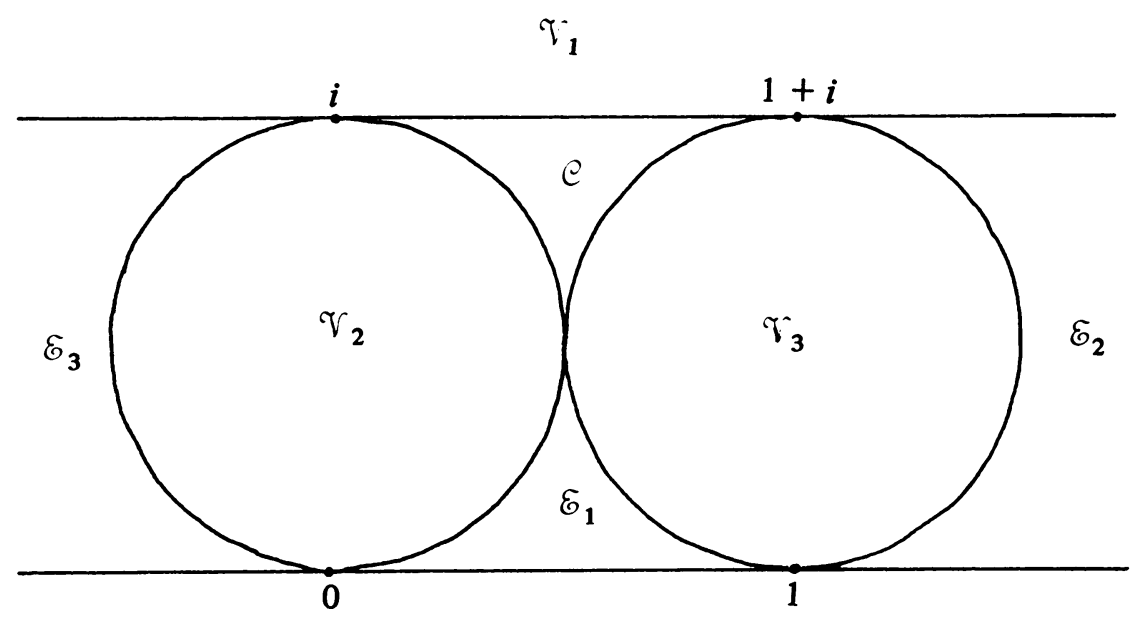

FiguRE 1. The seven basic Farey sets

Let $T_{F}$ denote the matrices corresponding to $t_{F}$ and $E_{F}=\left\{E_{1}, E_{2}, E_{3}\right\}$. A regular product is a product $M_{n}=T_{0} T_{1} \cdots T_{n}$ satisfying

(i) $T_{0}=V_{1}^{b_{0}}, b_{0} \in \mathbf{Z}, T_{1} \neq V_{1}$;

(ii) $T_{m} \in T_{F}$ if $\operatorname{det}\left(T_{0} T_{1} \cdots T_{m-1}\right)= \pm 1$;

(iii) $T_{m} \in T_{F} \backslash E_{F}$ if $\operatorname{det}\left(T_{0} T_{1} \cdots T_{m-1}\right)= \pm i$.

The notation $m_{n}$ or $M_{n}$ will refer to a regular product. It can be shown [3] that, for $m_{n} \in G_{p}, F\left(m_{n}\right)=m_{n}(\mathcal{T})$ and, for $m_{n} \in G_{i}, F\left(m_{n}\right)=m_{n}\left(\mathcal{T}^{*}\right)$.

The Farey sets $F\left(m_{n}\right)$ can be thought of as describing a structure of subdivisions of the complex plane. In particular, if $m_{n-1} \in G_{p}$, then $F\left(m_{n-1} \circ t_{n}\right)$, for $t_{n} \in t_{F}$, 
subdivides $F\left(m_{n-1}\right)$ into 3 circular Farey sets (the $\left.F\left(m_{n-1} \circ v_{j}\right)\right)$ and 4 triangular Farey sets (the $F\left(m_{n-1} \circ e_{j}\right)$ and $F\left(m_{n-1} \circ c\right)$ ). These sets intersect only on their boundaries, the 3 circles are pairwise tangent and tangent to $F\left(m_{n-1}\right)$ (which is circular). If $m_{n-1} \in G_{i}$, then $F\left(m_{n-1} \circ t_{n}\right)$, for $t_{n} \in t_{F} \backslash e_{F}$, defines a subdivision of $F\left(m_{n-1}\right)$ into 1 circular Farey set $\left(F\left(m_{n-1} \circ c\right)\right)$ and 3 triangular Farey sets (the $\left.F\left(m_{n-1} \circ v_{j}\right)\right)$. The set $F\left(m_{n-1} \circ c\right)$ is tangent to the 3 sides of $F\left(m_{n-1}\right)$.

The following two lemmas are found in [3]. The first uses the concept of the norm of a Farey set. Each Farey set $F(m)$ has an associated triple of points $m(\infty)$, $m(0)$, and $m(1)$ on its boundary, a Farey triangle. If $m$ has the matrix

$$
M=\left(\begin{array}{ll}
p_{1} & p_{2} \\
q_{1} & q_{2}
\end{array}\right)
$$

then these points are simply $p_{1} / q_{1}, p_{2} / q_{2}$, and $\left(p_{1}+p_{2}\right) /\left(q_{1}+q_{2}\right)=p_{3} q_{3}$, respectively. The norm of the Farey triangle is defined by $N(F T)=N\left(q_{1}\right)+N\left(q_{2}\right)+$ $N\left(q_{3}\right)$, where the $N$ on the right-hand side denotes the complex norm. The norm of a Farey set $F\left(m_{n}\right)$ is simply the norm of the associated Farey triangle (the more general $N(F(m))$ is somewhat more complicated).

LEMMA 1. If $m_{n}$ is regular, then

$$
\min \left\{N\left(F\left(m_{n} \circ t_{n+1}\right)\right)\right\}>N\left(F\left(m_{n}\right)\right)
$$

with equality iff $N\left(F\left(m_{n}\right)\right)=2$.

LEMMA 2. If $m_{n}$ is regular, then

$$
\operatorname{diam} F\left(m_{n}\right) \leqslant 4\left(N\left(F\left(m_{n}\right)\right)\right)^{-1 / 2}
$$

if $N>2$.

It follows from these lemmas that for an infinite sequence of Farey sets of the form $F\left(t_{0}\right) \supseteq F\left(t_{0} \circ t_{1}\right) \supseteq F\left(t_{0} \circ t_{1} \circ t_{2}\right) \supseteq \ldots$, if the norm 2 is ever exceeded, then the sequence has exactly one point in its intersection. Hence arises the correspondence between a number $\xi$ and a sequence of regular products.

Let $\mathscr{F}_{n}=\left\{F\left(m_{n}\right): m_{n}\right.$ is a regular product $\}$. The $\mathscr{F}_{n}$ are all disjoint, as is seen from the structure of subdivisions that they produce. The main result is that every Farey set is found in exactly one of the $\mathscr{F}_{n}$.

The following two lemmas are found in [2].

LEMMA 3. If $m \in G$ is given by $m: z \rightarrow\left(p_{1} z+p_{2}\right) /\left(q_{1} z+q_{2}\right)$, then the center and radius of $m(\mathbf{R})$ are given by

$$
c(m)=\frac{p_{1} \overline{q_{2}}-p_{2} \overline{q_{1}}}{q_{1} \overline{q_{2}}-q_{2} \overline{q_{1}}}, \quad r(m)=\frac{1}{i\left(q_{1} \overline{q_{2}}-q_{2} \overline{q_{1}}\right)} .
$$

It follows from Lemma 3 and the fact that the $p_{i}, q_{i}$ are integral that $m(\mathbf{R})$ intersects $\mathbf{R}$ in a point of tangency, or intersects in 2 points and $c(m) \in \mathbf{R}$, or else does not intersect $\mathbf{R}$. The following lemma characterizes the two intersection cases.

LEMMA 4. If $m \in G$ and $m(\mathbf{R})$ is tangent to $\mathbf{R}$, then $m \in G_{p}$. If $c(m) \in \mathbf{R}$, then $m \in G_{i}$.

The main result can now be proved. 
THEOREM. Every Farey set is in a unique $\mathscr{F}_{n}$; i.e. $\mathscr{F}=\cup_{n=0}^{\infty} \mathscr{F}_{n}$, where the union is a disjoint one.

Proof. Suppose $F=F(m)$ is a circular Farey set and $F \notin \mathscr{F}_{n}$ for any $n$. It can be assumed WLOG that $F \subset \mathcal{T}$, since if $F$ has a canonical representation, then $v_{1}^{b_{0}}(F)$ clearly does also. There exists an $n \in N_{0}$, and a finite sequence of Farey sets given by regular products, such that $F\left(t_{0}\right) \supset F\left(t_{0} \circ t_{1}\right) \supset F\left(t_{0} \circ t_{1} \circ t_{2}\right)$ $\supset \cdots \supset F\left(t_{0} \circ t_{1} \circ \cdots \circ t_{n}\right) \supseteq F$, and $F\left(t_{0} \circ t_{1} \circ t_{2} \circ \cdots \circ t_{n} \circ t_{n+1}\right) \supset F$ for any $t_{n+1} \in t_{F}$. This follows from Lemmas 1 and 2 and the nature of the regular product subdivisions. There is no collection of sets $F\left(m_{n} \circ t_{n+1}\right)$ (where $m_{n}=$ $\left.t_{0} \circ t_{1} \circ t_{2} \circ \cdots \circ t_{n}\right)$ whose union could be $F$. Hence $F$ must "overlap" one of the $F\left(m_{n} \circ t_{n+1}\right)$, in the sense that $\left(F \cup F\left(m_{n} \circ t_{n+1}\right)\right) \backslash\left(F \cap F\left(m_{n} \circ t_{n+1}\right)\right)$ is not contained in either $F\left(m_{n} \circ t_{n+1}\right)$ or $F$. In fact it must overlap a circular set $F\left(m_{n} \circ t_{n+1}\right)$, since every triangular set $F\left(m_{n} \circ t_{n+1}\right)$ shares its concave sides with circular sets. But then $m_{n+1}^{-1}\left(m_{n+1}(\mathcal{T})\right)=\mathcal{T}$ and $m_{n+1}^{-1}(m(\mathcal{T}))$ is a circular Farey set overlapping $\mathcal{T}$, which is ruled out by Lemma 4.

Suppose now that $F$ is triangular and assume WLOG that $F=m\left(\sigma^{*}\right)$. The sides of $F$ are arcs of $m \circ e_{j}(\mathbf{R}), j=1,2,3$, since the sides of $\mathcal{T}^{*}$ are arcs of $e_{j}(\mathbf{R})$. But $m \circ e_{j} \in G_{p}$, so the Farey sets $F\left(m \circ e_{j}\right)$ are all circular Farey sets, and hence have representations in terms of regular products. Let these three circular Farey sets be $F_{1}=\left(m_{n_{1}}\right), F_{2}=\left(m_{n_{2}}\right)$, and $F_{3}=\left(m_{n_{3}}\right)$, where $n_{1} \leqslant n_{2}<n_{3}$.

The $F_{j}$ determine 2 triangular regions. One is $F(m)=m\left(\mathscr{J}^{*}\right)$ and the other is $m\left(\overline{\mathfrak{T}}^{*}\right)$; the latter is not a Farey set, since $m\left(\overline{\mathfrak{T}}^{*}\right)$ determines $m(\overline{\mathfrak{T}})$, on which the choice between $m\left(\mathscr{T}^{*}\right)$ and $m\left(\overline{\mathcal{T}}^{*}\right)$ depends in the definition of a triangular Farey set. It follows from the subdivision process induced by regular maps that one of $m\left(\mathscr{J}^{*}\right)$ and $m\left(\overline{\mathfrak{T}}^{*}\right)$ is a triangular Farey set associated with a regular product $m_{n}$. Hence $F(m)=F\left(m_{n}\right)$, and so $F$ corresponds to a regular product. This completes the proof.

The content of this paper formed part of a doctoral thesis at the Claremont Graduate School. I would like to express appreciation to Professor William J. LeVeque, under whom my program was begun, to Professor Jerome Spanier, who provided a helpful push to complete the work, and to Professor Edward Thurber, who helped to clarify ideas.

\section{REFERENCES}

1. Lester R. Ford, Rational approximations to irrational complex numbers, Trans. Amer. Math. Soc. 19 (1918), 1-42.

2. Norman Richert, Diophantine approximation of complex numbers, unpublished thesis, Claremont Graduate School, 1980.

3. Asmus Schmidt, Diophantine approximation of complex numbers, Acta Math. 134 (1975), 1-85.

Department of Mathematics, Loyola Marymount University, Los Angeles, Calmornia 90045

Current address: Department of Mathematics, Statistics and Computer Science, Marquette University, Milwaukee, Wisconsin 53233 